\title{
Physiotherapy based on problem-solving in upper limb function and neuroplasticity in chronic stroke patients: A case series
}

\author{
Raquel Carvalho PT, MSc, Doctoral Student ${ }^{1,2}$ (D) । \\ Elsa Azevedo MD, PhD, Doctoral of Medicine ${ }^{3}$ (D) । Paulo Marques PhD, Eng ${ }^{2,4}$ (I) । \\ Nuno Dias PhD, Eng ${ }^{2,4,5}$ (D) । João José Cerqueira MD, PhD, Doctoral of Medicine M $^{2,4}$
}

${ }^{1}$ Department of Physical Therapy, CESPU, Institute of Research and Advanced Training in Health Sciences and Technologies, Portugal

${ }^{2}$ Life and Health Sciences Research Institute (ICVS), School of Health Sciences, University of Minho, Portugal

${ }^{3}$ Department of Neurology, Hospital São João and Faculty of Medicine of University of Porto, Portugal

${ }^{4}$ ICVS/3B's-PT Government Associate Laboratory, Braga/Guimarães, Portugal

${ }^{5}$ DIGARC, Polytechnic Institute of Cavado and Ave, Barcelos, Portugal

Correspondence

Raquel Carvalho, MSc, Doctoral Student, Departamento de Fisioterapia da Escola Superior de Saúde de Vale do Sousa (ESSVS), CESPU-IPSN, Rua Central de Gandra, 1317, 4585-116 Gandra, Paredes, Portugal. Email: raquel.carvalho@ipsn.cespu.pt

\begin{abstract}
Rationale, aims, and objectives: Upper limb recovery is one of the main concerns of stroke neurorehabilitation. Neuroplasticity might underlie such recovery, particularly in the chronic phase. The purpose of this study was to assess the effect of physiotherapy based on problem-solving in recovering arm function in chronic stroke patients and explore its neuroplastic changes.
\end{abstract}

Methods: A small sample research design with a $n$ of 3 using a pre-post test design was carried out. Neuroplasticity and function were assessed by using functional magnetic resonance imaging (during motor imagery and performance), action research arm test, motor assessment scale, and Fugl-Meyer assessment scale, at 3 sequential time periods: baseline(m0-before a 4-week period without physiotherapy), pretreatment(m1), and post-treatment(m2). Minimal clinical important differences and a recovery score were assessed. Assessors were blinded to moment assignment. Patients ${ }^{1}$ underwent physiotherapy sessions, 50 minutes, 5 days/week for 4 weeks. Four control subjects served as a reference for functional magnetic resonance imaging changes.

Results: All patients recovered more than $20 \%$ after intervention. Stroke patients had similar increased areas as healthy subjects during motor execution but not during imagination at baseline. Consequently, all patients increased activity in the contralateral precentral area after intervention.

Conclusions: This study indicates that 4 weeks of physiotherapy promoted the recovery of arm function and neuroplasticity in all chronic stroke patients. Future research is recommended to determine the efficacy of this therapy.

KEYWORDS

arm, Bobath concept, neuroplasticity, physiotherapy, rehabilitation, stroke

\section{I INTRODUCTION}

Upper limb function is affected in a great percentage of stroke patients; however, only a small fraction achieves complete recovery. ${ }^{1,2}$ Thus, recovery is a priority of neurorehabilitation. ${ }^{3}$

Rehabilitation and recovery after stroke are directed by neuroplasticity, ${ }^{3}$ ie, the ability of the brain to change its structure and 
intervention type, age, lesion location (cortical/subcortical or dominant/non dominant hemisphere), and handedness. ${ }^{7-9}$ Hence, there are many techniques, such as functional magnetic resonance imaging (fMRI), which researchers use to probe brain activity as well as the impact of neurorehabilitation strategies upon neuroplasticity. In this regard, fMRI is crucial because it has high spatial resolution, thus providing insights concerning the brain reorganization phenomena underlying functional recovery after neurorehabilitation. ${ }^{6}$

Motor rehabilitation traditionally focuses on conventional therapy, the most widespread being physiotherapy based on the Bobath Concept approach (PT). ${ }^{4,10-13}$ It attempts to integrate postural control and task performance with a specific focus on the quality of the latter, thus enhancing movement efficiency and quality. ${ }^{14}$ In practice, the key aspects of the Bobath clinical framework are the analysis of task performance, the relationship of posture and movement, and the role of sensory information in motor control. ${ }^{14}$ This approach focuses on neurophysiologic and motor learning theories which detect and analyse problems within functional activities and daily life participation, ${ }^{10}$ and assess movement components and primary impairments, improving efficiency and promoting a generalization in daily life. ${ }^{4}$ Repetition, functional goal-directed activities, and increased attention during learning are common strategies and can induce changes in cortical representation, hence neuroplasticity, which is use dependent. ${ }^{4,15}$

Despite these indicators and 3 systematic reviews, ${ }^{10,11,16}$ pointing to the effectiveness of this therapy, it is still controversial, particularly when considering data which reveals no effects of such intervention. ${ }^{16}$ These discrepancies can be due to the use of inappropriate outcome measures when assessing the improvement at motor level ${ }^{17}$ or due to the intervention procedures which do not reflect the current practice. ${ }^{12}$ Indeed, recent studies reveal that this approach has a similar efficacy compared with a constraint-induced therapy in patients with high functional levels ${ }^{18}$ while several others state the Bobath concept to be more effective than other therapeutic approaches (reviewed in Vaughan-Graham et $\mathrm{al}^{19}$ ). Nonetheless, PT is more inclusive and therefore can be applied to any patient no matter age or degree of functional severity, ${ }^{4.12}$

Consequently, this case series assessed the effect/impact of PT on the recovery of upper limb function in chronic stroke patients and explored its neuroplasticity changes.

\section{2 | MATERIAL AND METHODS}

\section{1 | Type of study}

A small sample research design with a $n$ of 3 using a pre-post test design was used.

\section{2 | Sample and setting}

Patients were recruited from the outpatient service at a Regional General Hospital after the Ethics Committee approved protocol. Patients with a first-ever left middle cerebral artery (MCA) episode, cortical infarcts $\geq 12$ months, were prospectively selected. Infarct site was identified using structural MR or CT scans and classified through clinic radiological criteria. Exclusion criteria involved hand plegia, persistent language and cognitive deficit (Mini Mental State Examination), major sensory deficits, inattention, visual impairment, depression, inability to toilet oneself independently prior to stroke, left-handedness (Edinburgh Handedness Inventory), severe spasticity (Modified Ashworth Spasticity Scale $\geq 3$ ), ${ }^{20}$ inability to perform accurate motor imagery, ie, a score below $75 \%$ in the hand rotation component (see Sharma et $\mathrm{al}^{21}$ ), and contraindications to MRI (eg, claustrophobia or pacemaker).

Four control subjects ( 2 males) were recruited through local advertisement to serve as a reference of $\mathrm{FMRI}$ changes and matched to cases regarding age, gender, and education. Additionally, control subjects had no history of medical disorders.

All subjects gave written consent according to the Declaration of Helsinki.

No dropouts or unpleasant experiences occurred with any assessment or therapy.

\section{3 | Procedures}

Each patient was assessed at 3 sequential time periods: 5 weeks before intervention (baseline (m0)-no PT administered); 1 week before initiating intervention (pre-treatment (m1)) to evaluate spontaneous recovery by time and to ensure stable baseline (control condition) and finally, 1 week after intervention (post-treatment (m2)-experimental condition), with a 4-week interval between each assessment.

Healthy subject outcomes were recorded at baseline and after a 4week interval ( $\mathrm{m} 0$ and $\mathrm{m} 1$, respectively).

\subsection{1 | Motor execution and imagination paradigm for fMRI}

FMRI assessed neuroplasticity (cortical activity and change of activation patterns) as a primary outcome measure. The fMRI paradigm, which was repeated 5 times, is a block design finger-thumb opposition sequence (30 seconds each), alternating motor imagery or motor execution (MI or ME) with a rest for each hand. The initiating hand was random (eg, MI_right/rest/ME_right/rest/MI_left/rest/ME_left/rest or MI_left/rest/ME_left/rest/MI_right/rest/ME_right/rest), and finger movements during scanning were monitored by using individual cameras and then registered based on the EMG.

\subsection{2 | FMRI data acquisition and processing}

Setting: Siemens 3.0 T (Siemens, Magnetom Vision, Erlangen, Germany), FoV: $245 \mathrm{~mm}^{2}$, spatial resolution: $3 \times 3 \times 3 \mathrm{~mm}^{3}$, TR 3 seconds, TE $30 \mathrm{~ms}$, flip angle: $90^{\circ}, 46$ slices. FMRI analysis was processed in Statistical Parametric Mapping software (SPM8-http://www.fil.ion.ucl. ac.uk/spm/) using a standard pre-processing pipeline (slice timing correction, realignment, coregistration of structural and functional images, normalization, and smoothing). Excessive head movement did not occur. Additionally, T1-weighted anatomical images with pulse sequence parameters of the entire brain were obtained: FoV $256 \mathrm{~mm}^{2}$; spatial resolution: $1 \times 1 \times 1.2 \mathrm{~mm}^{3}$; TR $=2300 \mathrm{~ms}$; $\mathrm{TE}=2.98 \mathrm{~ms}$; flip angle $9^{\circ} ; 160$ slices. 


\subsection{3 | Behavioural measure}

Motor impairment level and upper limb function were assessed by Action research arm test (ARAT), Fugl-Meyer assessment scale-arm section (FMA), and Motor assessment scale (MAS). ${ }^{22-24}$ The modified Rankin Scale (mRS) was used to measure the global/overall degree of disability/dependence. Each clinical score demonstrates good to excellent intra-rater variability, reliability, all valid in a stroke population. ${ }^{25}$ Assessment was performed and documented on video while 2 assessors (blind for assessment moment) scored behavioural outcome measures based on videotaped information at the end.

\section{4 | Intervention}

\subsection{1 | Physiotherapy based on the Bobath concept}

This approach usually takes 2 to 20 weeks, ${ }^{10,11,16}$ in a mean of $40 \mathrm{~min} /$ day during 20 to 24 sessions. Nonetheless, due to time management/ efficiency, PT involved 20 sessions of 50 minutes, 5 days/week, ${ }^{11,26}$ during 4 weeks. ${ }^{16,26}$ The therapist who performed all treatments had more than 10 years of experience in this therapy and has 1 basic course, 2 advanced courses, and several other thematic courses. The interventions were grouped in 5 major items: facilitated movement; mobilization; practicing an activity component; and/or whole task and teaching patients (see Tyson et $\mathrm{al}^{13}$ ). All interventions, individually programmed, aimed at improving postural control of trunk and shoulder girdle enabling more selective arm movement and increasing strength and function, ${ }^{12}$ to recover full motor range patterns, ${ }^{17}$ in order to enhance efficiency and maximize function and reducing the severity of impairments where they influence function. ${ }^{17}$ Tasks were previously known, simple with a functional goal not resembling the outcome measures, ie, tasks involved the facilitation of sitting to standing and/or walking, scapulohumeral rhythm, reaching partterns, prepararing for upper limb placing, selective strength training of the intrinsic muscles of the hand, among others. ${ }^{27}$ All interventions were administered at the patients' homes.

\section{5 | Data analysis}

Behavioural data, due to sample size, was analysed based on the minimal clinical important difference (MCID), representing the smallest amount of change in an outcome, which may be important to the patient or therapist. The MCID was set at $10 \%$ of the total range of the scales, except for FMA (scores between 4.25 and 7.25), based on previous studies and together with the patients. ${ }^{23,24,28}$ In order to account for the functional heterogeneity of patients at baseline, a recovery score for the behavioural outcome was calculated, ie, the sum of the differences between $\mathrm{m} 1$ and $\mathrm{m} 2$ for each score, divided by the total score at $\mathrm{m} 1$ (ie, the sum of all measures) (eg, ((FMAm2 - FMAm1) + (ARATm2 - ARATm1)) + (MASm2 - MASm1)) / (FMAm1 + ARATm1 + MASm1)). ${ }^{29}$ Based on the directionality of the changed scores, increased scores represented an improvement in all clinical tests.

Regarding the fMRI data, statistical analysis was performed using SPM8. For the first-level analysis, 5 conditions were modelled, namely rest periods, movement execution for right/affected (MER) and left/ unaffected hands (MEL), and movement imagination for right/affected (MIR) and left/unaffected hands (MIL) with 30-second duration. For this study, only contrasts representing $\mathrm{ME}$ and $\mathrm{MI}$ of the affected hand were considered (ie, MIR, MER), by contrasting the corresponding conditions with baseline condition. The regions of interest were based on the bilateral ME network: precentral and postcentral, supplementary motor area (SMA), parietal superior, paracentral, thalamus, putamen, pallidum, and cerebellum. The contrast estimate presented has a T/FStat threshold of $P<0.001$ combined with a minimum/critical cluster size of 20 voxels. These regions were defined by using the Automated Anatomic Labeling (AAL) atlas. ${ }^{30}$ The data are presented in reference to the side of cortical activity, using the terms contralateral/left or ipsilateral/right for all participants, which in stroke patients refers to left/ ipsilesional or right/contralesional cortex, respectively.

The differences between contrast estimate at different moments were also calculated subtracting the activation areas from $\mathrm{m} 1$ and $\mathrm{m} 0$ and between $\mathrm{m} 2$ and $\mathrm{m} 1$, with a T/F-Stat threshold of $P<0.001$, without a minimum cluster size.

\section{3 | RESULTS}

\section{1 | Case description}

Table 1 shows the main subject characteristics.

Three stroke patients were recruited aging from 43 to 72 , ( 1 male) with time from stroke onset ranged from 14 months to 37 months, and 4 healthy control subjects ( 2 males), aging from 48 to 74 (Table 1). The patients could walk outdoors (however, P2 needed to use a cane for 10-m distance), had familiar support, and were retired since stroke, due to their incapacity to do the usual tasks.

\subsection{Clinical and functional recovery}

Table 2 describes the MCID reference values of each functional assessment, baseline values, and gains after 4 weeks without any

TABLE 1 Patient characteristics according to age, gender, living situation, educational level and cognitive level, comorbidity, lesion location, time post stroke, and severity

\begin{tabular}{|c|c|c|c|c|c|c|c|c|c|c|}
\hline Group & & $\begin{array}{l}\text { Age } \\
\text { (Years) }\end{array}$ & $\begin{array}{l}\text { Gender } \\
(\mathrm{M} / \mathrm{F})\end{array}$ & $\begin{array}{l}\text { Living } \\
\text { Situation }\end{array}$ & $\begin{array}{l}\text { Educational } \\
\text { Level (Years) }\end{array}$ & MMSE & Comorbidity & $\begin{array}{l}\text { Lesion } \\
\text { Site }\end{array}$ & $\begin{array}{l}\text { Time from } \\
\text { Stroke (Months) }\end{array}$ & $\mathrm{mRS} \times / 6$ \\
\hline \multirow[t]{3}{*}{ Stroke } & $\mathrm{P} 1$ & 72 & $M$ & With family & 4 & 27 & --- & L MCA & 14 & 3 \\
\hline & $\mathrm{P} 2$ & 61 & $\mathrm{~F}$ & Alone & 4 & 29 & Knee arthrosis & L MCA & 37 & 4 \\
\hline & P3 & 43 & $\mathrm{~F}$ & With family & 12 & 28 & --- & L MCA & 18 & 2 \\
\hline \multirow[t]{4}{*}{ Healthy } & C1 & 66 & $M$ & With family & 4 & 29 & --- & --- & --- & --- \\
\hline & $\mathrm{C} 2$ & 74 & $M$ & With family & 4 & 28 & --- & --- & --- & --- \\
\hline & C3 & 63 & $\mathrm{~F}$ & Alone & 7 & 28 & Knee arthrosis & --- & --- & --- \\
\hline & C4 & 48 & $\mathrm{~F}$ & With family & 9 & 30 & --- & --- & --- & --- \\
\hline
\end{tabular}


TABLE 2 Functional outcome measures and respectively minimal clinically important differences (ARAT, MAS, and FMA) of each patient, differences/gains after 4 weeks without intervention $(\mathrm{m} 1-\mathrm{m} 0)$, with physiotherapy $(\mathrm{m} 2-\mathrm{m} 1)$ and, respectively, recovery score

\begin{tabular}{|c|c|c|c|c|}
\hline $\begin{array}{l}\text { Outcomes and MCID Reference } \\
\text { Value/ Total Scale Value }\end{array}$ & Moment & P1 & P2 & P3 \\
\hline ARAT $5.7( \pm 2$ sd) points $/ 57$ total & $\begin{array}{l}\text { Baseline } \\
\text { m1 - mo } \\
\text { m2 - m1 }\end{array}$ & $\begin{array}{l}29 \\
3 \\
1\end{array}$ & $\begin{array}{l}4 \\
4 \\
-1\end{array}$ & $\begin{array}{l}48 \\
-5 \\
8^{a}\end{array}$ \\
\hline MAS 1.8 points/18 total & $\begin{array}{l}\text { Baseline } \\
\mathrm{m} 1-\mathrm{m0} \\
\mathrm{m} 2-\mathrm{m} 1\end{array}$ & $\begin{array}{l}6 \\
2.5^{a} \\
2^{a}\end{array}$ & $\begin{array}{l}2 \\
-1 \\
1\end{array}$ & $\begin{array}{l}12 \\
1.5 \\
3^{\mathrm{a}}\end{array}$ \\
\hline Total recovery score (\%) & $\begin{array}{l}\mathrm{m} 1 \\
\mathrm{~m} 2\end{array}$ & $\begin{array}{l}22^{\mathrm{a}} \\
27^{\mathrm{a}}\end{array}$ & $\begin{array}{l}-1 \\
41^{a}\end{array}$ & $\begin{array}{l}-8 \\
20^{\mathrm{a}}\end{array}$ \\
\hline
\end{tabular}

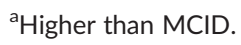

ARAT, Action research arm test; FMA, Fugl-Meyer assessment scale-arm section; MAS, Motor assessment scale; MCID, Minimal Clinically Important Difference; $\mathrm{m} 1$, moment 1 after 4 weeks without any intervention; $\mathrm{m} 1$ - m0, gains after 4 weeks without any intervention; $\mathrm{m} 2$, moment 2 after 4 weeks of intervention; $\mathrm{m} 2$ - $\mathrm{m} 1$, gains after 4-weeks of intervention.

intervention ( $\mathrm{m} 1-\mathrm{m} 0)$, after 4 weeks of PT (m2 - m1), ie, differences between each scale total score at $\mathrm{m} 1$ and $\mathrm{m} 0$ and $\mathrm{m} 2$ and $\mathrm{m} 1$, respectively, and the total recovery score.

During the non-intervention period, the female subjects (P2 and P3) exhibited stable or even decreased motor deficits, ie, below MCID. P1 improved MAS and FMA and kept improving after intervention (Table 2). After 4 weeks of PT, P1 showed improvement in 2 of 3 outcome measures, P2 only improved in FMA while P3 improved in all measures, being FMA the most significant for all patients (Table 2). The recovery score was higher after 4 weeks of intervention even in P1.

\section{3 | FMRI findings}

This section presents the main fMRI findings, which occurred cortically, ipsilateral/right, and/or contralateral/left to the hand movement or imagination.

\subsection{1 | Healthy and non-intervention stroke patient motor execution}

Table 3 depicts the areas where increased activity was observed, during ME of the right hand in comparison to rest, in healthy subjects (+)

TABLE 3 Motor execution activity compared with rest in healthy subjects and stroke patients at different moments (m0, $\mathrm{m} 1$, and $\mathrm{m} 2$ )

\begin{tabular}{|c|c|c|c|c|c|c|c|c|c|c|c|c|c|c|c|c|c|c|}
\hline \multirow[b]{2}{*}{ Region } & \multirow[b]{2}{*}{ Side } & \multicolumn{7}{|l|}{$\mathrm{mo}$} & \multicolumn{7}{|l|}{$\mathrm{m} 1$} & \multicolumn{3}{|l|}{$\mathrm{m} 2$} \\
\hline & & C1 & $\mathrm{C} 2$ & C3 & C4 & P1 & P2 & P3 & C1 & $\mathrm{C} 2$ & $\mathrm{C} 3$ & $\mathrm{C} 4$ & P1 & $\mathrm{P} 2$ & P3 & P1 & P2 & P3 \\
\hline Precentral & C & + & + & + & + & $x$ & $x$ & $x$ & + & + & + & + & $x$ & $x$ & $x$ & $x$ & $x$ & $x$ \\
\hline Precentral & I & + & + & + & + & & $x$ & $x$ & + & + & & + & $x$ & $x$ & $x$ & & $x$ & $x$ \\
\hline Postcentral & C & + & + & + & + & $x$ & $x$ & $x$ & + & + & + & + & $x$ & $x$ & $x$ & & $x$ & $x$ \\
\hline Postcentral & I & + & + & & + & & $x$ & $x$ & + & + & & + & $x$ & $x$ & $x$ & & $x$ & $x$ \\
\hline SMA & C & + & + & + & + & & $x$ & $x$ & + & + & + & + & & $x$ & $x$ & & $x$ & $x$ \\
\hline SMA & I & + & + & & + & & $x$ & $x$ & + & + & & & & $x$ & $x$ & & $x$ & $x$ \\
\hline Parietal superior & C & + & & & & & & $x$ & + & & & & & $x$ & $x$ & & $x$ & $x$ \\
\hline Parietal superior & I & + & & & & & & $x$ & + & & & & $x$ & $x$ & & & $x$ & \\
\hline Paracentral & C & & + & & & $x$ & & & & + & & & & $x$ & & & & \\
\hline Paracentral & I & & & & & & & & & & & & & & & & & \\
\hline Cerebellum_6 & C & + & + & & + & $x$ & $x$ & $x$ & + & + & + & + & & $x$ & $x$ & & $x$ & $x$ \\
\hline Cerebellum_6 & I & + & + & + & + & $x$ & $x$ & $x$ & + & + & + & + & & $x$ & $x$ & & $x$ & $x$ \\
\hline Cerebellum_8 & C & & & & & $x$ & $x$ & $x$ & & & & & & & $x$ & & $x$ & $x$ \\
\hline Cerebellum_8 & I & + & + & & + & $x$ & $x$ & $x$ & + & + & & + & & & $x$ & & $x$ & $x$ \\
\hline Thalamus & C & & + & + & + & & $x$ & $x$ & & + & & & $x$ & $x$ & & & $x$ & $x$ \\
\hline Thalamus & I & & + & & & & $x$ & & + & + & & & $x$ & $x$ & & & $x$ & $x$ \\
\hline Putamen & C & & & & + & & $x$ & & & + & & & $x$ & $x$ & & & $x$ & $x$ \\
\hline Putamen & 1 & & & & & & $x$ & & + & & & & $x$ & $x$ & & & $x$ & $x$ \\
\hline Pallidum & C & & & & + & & $x$ & & & & + & & & & & & & $x$ \\
\hline Pallidum & I & & & & + & & & & & & & & & & & & & $x$ \\
\hline
\end{tabular}

+ or $\mathrm{x}$-increased activation during right hand execution compared with rest, in healthy subjects or patients, respectively in the different moments (m0baseline; m1-4/wk interval/pre-intervention; m2-post-intervention), with a T/F-Stat threshold of $P<0.001$ (minimum cluster size of 20 voxels).

Abbreviations: C, contralateral; I, ipsilateral. 
and stroke patients $(\mathrm{x})$, at baseline, after a 4-week interval and postintervention.

At baseline ( $\mathrm{m} 0$ ), all healthy subjects showed increased activity in bilateral precentral. Moreover, increased activity in bilateral postcentral, SMA, and cerebellum_6 and right cerebellum_8 was also observed, except in C3. P2 and P3 had the same behavior at baseline in precentral, postcentral, SMA, and cerebellum_6; however, the activity in cerebellum_8 was bilateral. C1 also increased activity in the bilateral parietal superior area. C3 as well as P1 demonstrated more contralateral cortical activity. The activation in basal ganglia was more variable.

After a 4-week interval, the areas where increased activation was observed in healthy subjects remained similar but with some differences in terms of intensity of contrast estimate and volumes. In general, healthy subjects, 3 out of 4 increased their contrast estimate intensity (except for C4). All healthy subjects increased left precentral area activity; however, while men increased volume/area, women decreased, ie, focused activation. The augmented activation from the ipsilateral cerebellum 6 and contralateral postcentral areas was observed in 3 out 4 healthy subjects, ie, despite the increased activity of left precentral area, women also increased activity in both right cerebellum 6 and 8 (C3) and left postcentral (C4). Notwithstanding, there was decreased activity from $\mathrm{m} 0$ to $\mathrm{m} 1$ in all areas at $\mathrm{m} 0$ in both C3 and C4. However, men had different behavior, not only compared with women but also between them; C1 increased activity, while C2 decreased. Both $\mathrm{C} 1$ and $\mathrm{C} 2$ increased bilateral postcentral, right cerebellum 6 and left putamen but C2 decreased activity in left cerebellum 6, right thalamus and bilateral paracentral. Thus, a quite heterogeneous pattern.

Similar to healthy subjects, P1 increased brain activity intensity between $\mathrm{m} 0$ and $\mathrm{m} 1$. Contrarily, P2 and P3 decreased it. No differences between moments were found during a 4-week interval without intervention ( $\mathrm{m} 1-\mathrm{m} 0$ ) in contralateral precentral area; however, all patients presented increased activity in other areas such as, postcentral ( $\mathrm{P} 1$ bilateral while $\mathrm{P} 2$ and $\mathrm{P} 3$ contralateral) and parietal superior (P1 contralateral while P2 and P3 bilateral). P1 also increased activity in bilateral thalamus and putamen, while P2 increased in right thalamus and cerebellum_8. Like healthy subjects, P1 increased while P2 and P3 decreased volume/area.

There was decreased activity in all patients in postcentral (P1 contralateral while P2 and P3 bilateral), SMA (P1 and P2 contralateral while P3 ipsilateral), cerebellum_6 (P1 contralateral while P2 and P3 bilateral), and cerebellum_8 (P1 and P2 bilateral while P3 contralateral). Moreover, there was also decreased activity in the contralateral and bilateral precentral areas, in P2 and P3, respectively.

\subsection{2 | Healthy and non-intervention stroke patient motor imagination}

Table 4 illustrates the areas where increased activity was observed, during motor imagination of right hand in comparison to rest, in healthy subjects $(+)$ and stroke patients $(x)$ according to the moment.

In healthy subjects, similarly to ME, all subjects showed, at baseline $(\mathrm{m} 0)$, increased activity in bilateral precentral and SMA, and except for $\mathrm{C} 3$, an increased activity in bilateral postcentral was observed.

TABLE 4 Motor imagination activity compared with rest in healthy subjects and stroke patients at different moments ( $\mathrm{m} 0$, $\mathrm{m} 1$, and $\mathrm{m} 2$ )

\begin{tabular}{|c|c|c|c|c|c|c|c|c|c|c|c|c|c|c|c|c|c|c|}
\hline \multirow[b]{2}{*}{ Region } & \multirow[b]{2}{*}{ Side } & \multicolumn{7}{|l|}{$\mathrm{mo}$} & \multicolumn{7}{|l|}{$\mathrm{m} 1$} & \multicolumn{3}{|l|}{$\mathrm{m} 2$} \\
\hline & & C1 & $\mathrm{C} 2$ & $\mathrm{C} 3$ & $\mathrm{C} 4$ & P1 & P2 & P3 & C1 & $\mathrm{C} 2$ & $\mathrm{C} 3$ & $\mathrm{C} 4$ & P1 & P2 & P3 & P1 & P2 & P3 \\
\hline Precentral & $\mathrm{C}$ & + & + & + & + & & & $x$ & + & + & + & + & & & $x$ & & & \\
\hline Precentral & I & + & + & + & + & & & $x$ & + & + & + & + & & & $x$ & & & $x$ \\
\hline Postcentral & $\mathrm{C}$ & + & + & & + & & & & + & + & + & + & & & $x$ & & & \\
\hline Postcentral & I & + & + & & + & & & & + & & & + & & & $x$ & & & \\
\hline SMA & $\mathrm{C}$ & + & + & + & + & & & $x$ & + & + & + & + & & & $x$ & & & \\
\hline SMA & I & + & + & + & + & & & $x$ & + & + & + & + & & & $x$ & & & \\
\hline Parietal superior & C & & + & + & + & & & & + & & + & + & & $x$ & & & $x$ & \\
\hline Parietal superior & I & & + & + & & & & & + & + & + & & & & & & $x$ & \\
\hline Paracentral & C & & & & & & & & & & & & & & & & & \\
\hline Paracentral & 1 & & & & & & & & & & & & & & & & & \\
\hline Cerebellum_6 & C & & + & + & + & & & & & & + & + & & & $x$ & & & \\
\hline Cerebellum_6 & I & & + & + & + & & & & & & + & + & & & $x$ & & & \\
\hline Cerebellum_8 & C & & & & & & & & & & & & & & & & & \\
\hline Cerebellum_8 & I & & + & + & & & & & & & + & & & & & & & \\
\hline Thalamus & $\mathrm{C}$ & & + & + & + & & & & & & + & + & & & & & $x$ & \\
\hline Thalamus & 1 & & + & & & & & & & & + & & & & & & & \\
\hline Putamen & C & & + & + & & & & & & & + & & & & & & & \\
\hline Putamen & I & & & & & & & & & & & & & & & & & \\
\hline Pallidum & C & & & + & & & & & & & + & & & & & & & \\
\hline Pallidum & I & & & + & & & & & & & + & & & & & & & \\
\hline
\end{tabular}

+ or $\mathrm{x}$-increased activation during right hand imagination compared with rest, in healthy subjects or patients, respectively in the different moments (m0baseline; m1 pre-intervention; m2-post-intervention), with a T/F-Stat threshold of $P<0.001$ (minimum cluster size of 20 voxels).

Abbreviations: C, contralateral; I, ipsilateral. 
Three of 4 subjects (ie, except $\mathrm{C} 1$ ) showed increased activity in bilateral cerebellum_6, in parietal superior (C2 and C3-bilateral or C4-contralateral) and in thalamus (C2 bilateral and C3 and C4 contralateral). Comparing to MER at baseline, intensity was relatively lower during MIR except for $\mathrm{C} 2$, who had quite similar behavior, not only in intensity but also in regions.

At baseline, 2 out of 3 patients were unable to increase activity comparatively with rest at baseline (P1 and P2), while P3 increased bilateral activity in the precentral and SMA areas.

All healthy subjects exhibited increased activity in the bilateral precentral area after a 4-week interval. However, the behavior was very different in each subject in the remaining areas, not only in which specific areas and tendency (increase or decrease) but also in intensity (preserved, decreased, or increased). C1 only increased activity (despite the mentioned area also increasing in the bilateral postcentral and parietal superior and ipsilateral SMA). C2 decreased activity and intensity in all the areas he had previously increased at m0. Both C3 and C4 decreased in contralateral SMA; however, C3 also decreased in contralateral precentral and right cerebellum_6. Besides, there was increased activity in bilateral parietal superior and cerebellum_ 6 in subject C3 and, in contralateral postcentral and cerebellum_6 in subject C4. Comparing to baseline, C3 and C4 increased contrast estimate intensity.

After reassessment, $\mathrm{P} 1$ maintained the tendency at $\mathrm{m0}$, but $\mathrm{P} 2$ was able to increase activity in contralateral/ipsilesional parietal superior. Moreover, owing to the reduction in volume/area, P3 increased activity in bilateral precentral and cerebellum_6, and in contralateral/ ipsilesional postcentral but decreased in contralateral/ipsilesional precentral.

\subsection{3 | Effect of physiotherapy on stroke patients}

After a 4-week intervention period, all patients increased contralateral/ipsilesional precentral activity during ME. Besides this, P2 and P3 increased activity in the ipsilateral/contralesional precentral and in bilateral postcentral areas, cerebellum_6 and 8, putamen, pallidum, and thalamus. P2 also increased activity in bilateral parietal superior areas. Relating to intensity, P1 maintained the same when comparing the differences between moments, while P2 and P3 increased more than double. There was a decreased activity in postcentral in all patients (P1 and P3 bilateral, P2 ipsilateral), in bilateral parietal superior (P2 and P3), and in bilateral SMA (P3).

During imagination, behavior was totally different among subjects; for P1 there was no change in the brain activity comparing to rest, P2 increased in contralateral/ipsilesional parietal superior and SMA, while P3 increased the ipsilateral/contralesional precentral area, and decreased bilateral precentral, postcentral, and cerebellum_6 comparing to $\mathrm{m} 1$.

\section{4 | DISCUSSION}

The present paper, a pre post-test small sample research design $(n=3)$, was conducted to assess the impact of Bobath concept on motor network plasticity in patients with chronic cortical left MCA stroke. Notwithstanding our small sample size, patients showed an improvement greater than the minimal clinically important difference and in the total recovery score after intervention, suggesting the benefit of intervention. Despite the different outcome measures used, functional gains are consistent with other studies, ${ }^{18,31}$ ie, intervention based on Bobath concept improves at least 1 functional measure. ${ }^{18,31,32}$ This is striking, considering these chronic patients for whom spontaneous recovery is minimal, ${ }^{5}$ obtained results within a relatively short timeframe of 4 weeks. $^{33}$ Thus, the data suggest motor deficits in such patients are amenable to PT suggesting more prolonged intervention may in fact induce greater recovery.

Interestingly, even with chronic stroke, 2 out of 3 patients ( $\mathrm{P} 2$ and P3) had a worsening of their upper limb function during the period of non-intervention. Although P1 improved, the recovery score was even higher after intervention (22\% comparing with $27 \%$ ), and when considering FMA, the result doubled in the same period.

Regarding fMRI findings, in healthy subjects, similarly to other studies, imagined or executed movements have the participation of the same cortical areas, ${ }^{34-37}$ with additional increased activity in precentral and postcentral areas ${ }^{34,38-40}$ yet, the magnitude and volume of brain activation are more limited during MIR compared with MER, in healthy subjects. ${ }^{21}$ The ipsilateral hemisphere (primary motor cortex [M1], premotor area, primary sensory cortex, and SMA) and bilateral cerebellar activation during performance of complex movement was previously mentioned in healthy subjects, ${ }^{41}$ which is consistent with our results. Consequently, the recruitment of several secondary areas in both healthy subjects and patients during MER and MIR can be related to the task itself. The increased activity in SMA comparably to rest, not only during MER but also during MIR, can be related to its facilitation effect in distal muscles, ${ }^{42}$ ie, it seems to be also guided throughout the task.

Stroke patients have similar behavior to healthy subjects at baseline, in contralateral/ipsilesional precentral and post central area and ipsilateral/contralesional cerebellum, during MER but quite different during MIR. In patients, the bilateral activation was quoted as a recruitment of the unaffected hemisphere to complete the task. ${ }^{37}$ Nonetheless, our data suggest that patients with cortical stroke had different abilities to perform motor imagination compared with healthy volunteers, because stroke may have damaged the intra-cortical circuitry, affecting cortical reorganization to compensate for functional deficits. ${ }^{43}$ Although $\mathrm{Ml}$ ability was assessed in all patients, age may have interfered. Indeed, the ability to mentally simulate upper-limb movements has been shown to decline with age, ${ }^{44}$ justifying differences between the inability of P1 and ability of P3 to perform MI. However, according to gender, the results were unexpected, because males seem to facilitate MI, possibly due to gender differences in visuospatial/ visuomotor processing, ${ }^{45,46}$ suggesting age having greater impact on MI ability.

After a 4-week interval, healthy subjects kept increasing activity in contralateral precentral compared with mo. Although our patients were not successful in this initial period, they were after a 4-week intervention. The fact that there is less task related contralateral/ ipsilesional activity during a non-intervention period, and increased after intervention, compared with baseline, was previously discussed, in patients with greater impairment. ${ }^{42}$ The decrease in cortical activation induced by $\mathrm{ME}$ between first $(\mathrm{m} 0)$ and second $(\mathrm{m} 1)$ assessments 
likely reflects a test-retest effect, probably due to task learning ${ }^{47}$ or training. ${ }^{48}$ Nonetheless, our results during ME had a different magnitude (lower contrast estimate intensity) in patients compared with controls, suggesting its mechanisms (ie, susceptibility to training/motor learning) can be affected in such patients.

A previous study revealed similar decreases in brain activation across multiple scan sessions, but only in recent patients and not controls, ${ }^{49}$ signifying overactivation in primary and secondary motor areas at baseline, ${ }^{50}$ which seems to be present in the chronic phase. Two different patterns of brain activation changed, ie, increased or decreased volume and the uniform or mixed patterns, were previously mentioned in stroke patients, after intervention, ${ }^{51}$ similar to what occurred in this study.

The previously mentioned and our results suggest that P1 probably performed some tasks (beyond physiotherapy) during the nonintervention period. The reduction in areas at $\mathrm{m} 2$ focusing on precentral area could be related to the improvement, similar to pruning during neurodevelopment, ${ }^{42}$ although the opposite occurred in P2 and P3, which might be characteristic of cortical stroke patients or gender.

Well-recovered hand function relying mostly on ipsilesional/contralateral activity is generally accepted ${ }^{52}$; therefore, the increase in contralateral/ipsilesional precentral area could justify the augmented function in P2 and P3 at m2 and the higher increase in P1. Nonetheless, some controversy exists regarding the role of contralateral/ ipsilesional M1, the current deficit, and patient improvement. Some authors have mentioned that recruiting contralateral/ipsilesional M1 differs across patients in a way largely independent to the baseline function or deficit. ${ }^{52}$ While, more recently, others refer there are differences in fMRI changes regarding severity at baseline, ie, a decrease or increase in brain activation occurs according to better or worse motor function for proximal arm at baseline, respectively, especially in contralateral M1 and ipsilateral SMA which is independent of functional improvement. ${ }^{51}$ This suggest that the variability in our results was the consequence of our patient characteristics, ie, none had language or cognitive deficits needed to understand instructions; the severity at baseline, because some studies mentioned the strategy to recovery is different and age. An increase in contralateral/ipsilesional or decrease/contralesional in ipsilateral hemisphere seems to be different strategies to improve function.

The remaining bilateral increased and decreased activity observed in P2 and P3 could be justified by "new" closed-loop circuit between the cerebellum and the postcentral area, because the somatosensory cortex (located in the postcentral area) contributes to the corticospinal tract. ${ }^{53}$ Because SMA seems to be bilaterally more activated in badrecoverees so it can be the necessary strategy to induce higher posttreatment function in severe patients. The decreased activation in P3 can be related to her recovery, because SMA activity seems to be a compensation to assist M1. ${ }^{54}$

Both P2 and P3 increased ipsilateral activity, previously correlated with poor outcome in subcortical stroke; ${ }^{52}$ however, because both patients improved upper limb function, this can be related to the use of the unaffected hemisphere, especially ipsilateral/contralesional premotor cortex because it has a potential and essential role in post-stroke rehabilitation. ${ }^{42,52}$ Hence, the ipsilateral/contralesional hemisphere activation observed in our study could be related to a new functional architecture of the brain after cortical stroke. This new motor system reorganization may not be as effective as an undamaged brain but an alternative to producing a more effective movement, in these particular cases, more function. However, this can also be observed after normal aging, ie, a recruitment of additional areas, in particular in the ipsilateral/contralesional hemisphere, ${ }^{42}$ justifying the similar results in healthy subjects. The success of recovery in chronic patients involved the recruitment of alternative single or multiple functional brain regions in contralateral/ipsilesional and ipsilateral/contralesional hemispheres. ${ }^{51}$

It has been shown that stroke patients with lesions of the precentral cortex, such as the ones in the present study, have increased connectivity between the cerebellum and intact perilesional areas ${ }^{54}$ and that a synergistic action of both regions is essential for the performance and learning of motor skills. ${ }^{48}$ More importantly, recovery of hand function after acute stroke has been directly correlated with increased activation of the ipsilateral cerebellum, and not with any other area, ${ }^{55,56}$ further supporting the significance of our findings.

Our most disabled patient (P2) is the one who recruited more regions, which is in line with other studies mentioning greater injury to corticospinal systems correlated with greater recruitment of secondary motor areas. ${ }^{42,51}$ This patient also had a better recovery score after intervention, coinciding with some authors, who claim greater motor function gains obtained by severe patients at baseline. ${ }^{51}$ This increased activity can be interpreted as a compensatory mechanism that, when encountering a lesion, contributes to the maintenance of its functions by recruiting additional areas. ${ }^{7}$ Thus, suggesting strokeinduced changes, in brain networks, are not exclusive to lesioned regions. The causes and consequences of such changes certainly merit consideration in future studies.

Our results are overall encouraging, although this study has several limitations, small sample size, which may have made us consider some changes as non-significant despite the fact that they may be significant. This study would benefit from the inclusion of a follow-up phase post-intervention that would strengthen it. However, we designed it as a case series, the conclusions of which should be further explored and detailed in subsequent trials. Additionally, some of our regions of interest were severely damaged by the infarct, directly affecting BOLD signals, and likely justifying the difficulty in observing changes in other areas with enough statistical power.

Despite these limitations, we are confident that our main findings are robust enough to be considered, highlighting the potential for rehabilitation and neuroplasticity in chronic stroke patients.

Physiotherapy based on the Bobath concept approach throughout 4 weeks increased both the contralateral/ipsilesional precentral area activity and upper limb function in the chronic stroke patients from this study. Nevertheless, further research is needed so that an effective form of post-stroke rehabilitation may be considered.

\section{DECLARATION OF INTEREST}

The authors declare that they have no conflict of interest. 


\section{ORCID}

Raquel Carvalho (D) http://orcid.org/0000-0002-7946-7708

Elsa Azevedo (i) http://orcid.org/0000-0003-3731-4508

Paulo Marques (D) http://orcid.org/0000-0002-6304-4989

Nuno Dias (1) http://orcid.org/0000-0002-0498-4612

João José Cerqueira (D) http://orcid.org/0000-0003-3155-2775

\section{REFERENCES}

1. Kwakkel G, Kollen BJ, van der Grond J, Prevo AJ. Probability of regaining dexterity in the flaccid upper limb: impact of severity of paresis and time since onset in acute stroke. Stroke. 2003;34(9):2181-2186.

2. Williams BK, Galea MP, Winter AT. What is the functional outcome for the upper limb after stroke? Aust J Physiother. 2001;47(1):19-27.

3. Hara Y. Brain plasticity and rehabilitation in stroke patients. J Nippon Med Sch. 2015;82(1):4-13.

4. Graham JV, Eustace C, Brock K, Swain E, Irwin-Carruthers S. The Bobath concept in contemporary clinical practice. Top Stroke Rehabil. 2009;16(1):57-68.

5. Taupin P. Adult neurogenesis and neuroplasticity. Restor Neurol Neurosci. 2006;24(1):9-15.

6. Hodics T, Cohen LG, Cramer SC. Functional imaging of intervention effects in stroke motor rehabilitation. Arch Phys Med Rehabil. 2006;87(12 Suppl 2):S36-S42.

7. Carey LM, Seitz RJ. Functional neuroimaging in stroke recovery and neurorehabilitation: conceptual issues and perspectives. Int J Stroke. 2007;2(4):245-264

8. Ward NS, Swayne OB, Newton JM. Age-dependent changes in the neural correlates of force modulation: an fMRI study. Neurobiol Aging. 2008;29(9):1434-1446.

9. Zemke AC, Heagerty PJ, Lee C, Cramer SC. Motor cortex organization after stroke is related to side of stroke and level of recovery. Stroke. 2003;34(5):e23-e28.

10. Kollen BJ, Lennon S, Lyons B, et al. The effectiveness of the Bobath concept in stroke rehabilitation: what is the evidence? Stroke. 2009;40(4):e89-e97.

11. Luke C, Dodd KJ, Brock K. Outcomes of the Bobath concept on upper limb recovery following stroke. Clin Rehabil. 2004;18(8):888-898.

12. Vaughan-Graham J, Cott C, Wright FV. The Bobath (NDT) concept in adult neurological rehabilitation: what is the state of the knowledge? A scoping review. Part I: conceptual perspectives. Disabil Rehabil. 2015;37(20):1793-1807.

13. Tyson SF, Connell LA, Busse ME, Lennon S. What is Bobath? A survey of UK stroke physiotherapists' perceptions of the content of the Bobath concept to treat postural control and mobility problems after stroke. Disabil Rehabil. 2009;31(6):448-457.

14. Vaughan-Graham J, Cott C. Defining a Bobath clinical framework-a modified e-Delphi study. Physiother Theory Pract. 2016;32(8):612-627.

15. Arya KN, Pandian S, Verma R, Garg RK. Movement therapy induced neural reorganization and motor recovery in stroke: a review. J Bodyw Mov Ther. 2011;15(4):528-537.

16. Paci M. Physiotherapy based on the Bobath concept for adults with post-stroke hemiplegia: a review of effectiveness studies. J Rehabil Med. 2003;35(1):2-7.

17. Levin MF, Panturin E. Sensorimotor integration for functional recovery and the Bobath approach. Motor Control. 2011;15(2):285-301.

18. Huseyinsinoglu BE, Ozdincler AR, Krespi Y. Bobath concept versus constraint-induced movement therapy to improve arm functional recovery in stroke patients: a randomized controlled trial. Clin Rehabil. 2012;26(8):705-715.

19. Vaughan-Graham J, Cott C, Wright FV. The Bobath (NDT) concept in adult neurological rehabilitation: what is the state of the knowledge? A scoping review. Part II: intervention studies perspectives. Disabil Rehabil. 2015;37(21):1909-1928.
20. Page SJ, Levine P, Khoury JC. Modified constraint-induced therapy combined with mental practice: thinking through better motor outcomes. Stroke. 2009;40(2):551-554.

21. Sharma N, Simmons LH, Jones PS, et al. Motor imagery after subcortical stroke: a functional magnetic resonance imaging study. Stroke. 2009;40(4):1315-1324.

22. Carr JH, Shepherd RB, Nordholm L, Lynne D. Investigation of a new motor assessment scale for stroke patients. Phys Ther. 1985;65(2):175-180.

23. Page SJ, Fulk GD, Boyne P. Clinically important differences for the upper-extremity Fugl-Meyer scale in people with minimal to moderate impairment due to chronic stroke. Phys Ther. 2012;92(6):791-798.

24. Yozbatiran N, Der-Yeghiaian L, Cramer SC. A standardized approach to performing the action research arm test. Neurorehabil Neural Repair. 2008;22(1):78-90.

25. Croarkin E, Danoff J, Barnes C. Evidence-based rating of upper-extremity motor function tests used for people following a stroke. Phys Ther. 2004;84(1):62-74.

26. Wang RY, Chen HI, Chen CY, Yang YR. Efficacy of Bobath versus orthopaedic approach on impairment and function at different motor recovery stages after stroke: a randomized controlled study. Clin Rehabil. 2005;19(2):155-164.

27. Raine S, Meadows L, Lynch-Ellerington M. Bobath Concept: Theory and Clinical Practice in Neurological Rehabilitation. West Sussex: WileyBlackwell; 2009.

28. Chen HM, Chen CC, Hsueh IP, Huang SL, Hsieh CL. Test-retest reproducibility and smallest real difference of 5 hand function tests in patients with stroke. Neurorehabil Neural Repair. 2009;23(5):435-440.

29. Westlake KP, Hinkley LB, Bucci M, et al. Resting state alpha-band functional connectivity and recovery after stroke. Exp Neurol. 2012;237(1):160-169.

30. Tzourio-Mazoyer N, Landeau B, Papathanassiou D, et al. Automated anatomical labeling of activations in SPM using a macroscopic anatomical parcellation of the MNI MRI single-subject brain. Neuroimage. 2002;15(1):273-289.

31. Langhammer B, Stanghelle JK. Can physiotherapy after stroke based on the Bobath concept result in improved quality of movement compared to the motor relearning programme. Physiother Res Int. 2011;16(2):69-80.

32. Timmermans AA, Verbunt JA, van Woerden R, Moennekens M, Pernot $\mathrm{DH}, \mathrm{Ha}$ S. Effect of mental practice on the improvement of function and daily activity performance of the upper extremity in patients with subacute stroke: a randomized clinical trial. J Am Med Dir Assoc. 2013;14(3):204-212.

33. Page SJ, Szaflarski JP, Eliassen JC, Pan H, Cramer SC. Cortical plasticity following motor skill learning during mental practice in stroke. Neurorehabil Neural Repair. 2009;23(4):382-388.

34. Weiss T, Hansen E, Beyer L, et al. Activation processes during mental practice in stroke patients. Int J Psychophysiol. 1994;17(1):91-100.

35. Page SJ, Levine $P$, Leonard A. Mental practice in chronic stroke: results of a randomized, placebo-controlled trial. Stroke. 2007;38(4):12931297.

36. Seitz RJ, Matyas TA, Carey LM. Neural plasticity as a basis for motor learning and neurorehabilitation. Brain Impairment. 2008;9(2):103-113.

37. Butler AJ, Page SJ. Mental practice with motor imagery: evidence for motor recovery and cortical reorganization after stroke. Arch Phys Med Rehabil. 2006;87(12 Suppl 2):S2-S11.

38. Lacourse MG, Orr EL, Cramer SC, Cohen MJ. Brain activation during execution and motor imagery of novel and skilled sequential hand movements. Neuroimage. 2005;27(3):505-519.

39. Hanakawa T, Dimyan MA, Hallett M. Motor planning, imagery, and execution in the distributed motor network: a time-course study with functional MRI. Cereb Cortex. 2008;18(12):2775-2788.

40. Jeannerod M. Mental imagery in the motor context. Neuropsychologia. 1995;33(11):1419-1432. 
41. Park JW, Kwon YH, Lee MY, et al. Brain activation pattern according to exercise complexity: a functional MRI study. NeuroRehabilitation. 2008;23(3):283-288.

42. Ward N. Assessment of cortical reorganisation for hand function after stroke. J Physiol. 2011;589(Pt 23):5625-5632.

43. Luft AR, Waller $S$, Forrester $L$, et al. Lesion location alters brain activation in chronically impaired stroke survivors. Neuroimage. 2004;21(3):924-935.

44. Saimpont A, Pozzo T, Papaxanthis C. Aging affects the mental rotation of left and right hands. PLoS One. 2009;4(8):e6714.

45. Schuster C, Hilfiker R, Amft O, et al. Best practice for motor imagery: a systematic literature review on motor imagery training elements in five different disciplines. BMC Med. 2011;9(1):75.

46. Schaechter JD, Perdue KL. Enhanced cortical activation in the contralesional hemisphere of chronic stroke patients in response to motor skill challenge. Cereb Cortex. 2008;18(3):638-647.

47. Rossini PM, Altamura C, Ferreri F, et al. Neuroimaging experimental studies on brain plasticity in recovery from stroke. Eura Medicophys. 2007;43(2):241-254.

48. Dong Y, Winstein CJ, Albistegui-DuBois R, Dobkin BH. Evolution of FMRI activation in the perilesional primary motor cortex and cerebellum with rehabilitation training-related motor gains after stroke: a pilot study. Neurorehabil Neural Repair. 2007;21(5):412-428.

49. Ward NS, Brown MM, Thompson AJ, Frackowiak RS. Neural correlates of motor recovery after stroke: a cross-sectional fMRI study. Brain. 2003;126(6):1430-1448.

50. Ward NS, Brown MM, Thompson AJ, Frackowiak RS. Neural correlates of motor recovery after stroke: a longitudinal fMRI study. Brain. 2003;126(Pt 11):2476-2496.
51. Pundik S, McCabe JP, Hrovat K, et al. Recovery of post stroke proximal arm function, driven by complex neuroplastic bilateral brain activation patterns and predicted by baseline motor dysfunction severity. Front Hum Neurosci. 2015;9:394.

52. Calautti C, Baron JC. Functional neuroimaging studies of motor recovery after stroke in adults: a review. Stroke. 2003;34(6):1553-1566.

53. Caligiore D, Pezzulo G, Baldassarre G, et al. Consensus paper: towards a systems-level view of cerebellar function: the interplay between cerebellum, basal ganglia, and cortex. Cerebellum. 2017;16(1):203-229.

54. Mintzopoulos D, Astrakas LG, Khanicheh A, et al. Connectivity alterations assessed by combining $\mathrm{fMRI}$ and MR-compatible hand robots in chronic stroke. Neuroimage. 2009;47(Suppl 2):T90-T97.

55. Small SL, Hlustik P, Noll DC, Genovese C, Solodkin A. Cerebellar hemispheric activation ipsilateral to the paretic hand correlates with functional recovery after stroke. Brain. 2002;125(Pt 7):1544-1557.

56. Rosso C, Valabregue R, Attal Y, et al. Contribution of corticospinal tract and functional connectivity in hand motor impairment after stroke. PLoS One. 2013;8(9):e73164.

How to cite this article: Carvalho R, Azevedo E, Marques P, Dias N, Cerqueira JJ. Physiotherapy based on problem-solving in upper limb function and neuroplasticity in chronic stroke patients: A case series. J Eval Clin Pract. 2018;1-9. https:// doi.org/10.1111/jep.12921 